\title{
Regulatory considerations for prospective patient care registries: lessons learned from the National Neurosurgery Quality and Outcomes Database*
}

\author{
Anthony L. Asher, M.D., ${ }^{1,2}$ Matthew J. McGirt, M.D., ${ }^{3}$ Steven D. Glassman, M.D. ${ }^{4}$ \\ Rachel Groman, M.P.H., ${ }^{5}$ Dan K. Resnick, M.D., M.S., ${ }^{6}$ Melissa MehrliCh, R.N., B.S.N., ${ }^{1}$ \\ Elizabeth Spivey, B.S., ${ }^{1}$ and Paul McCormick, M.D., M.P.H. ${ }^{7}$
}

${ }^{1}$ Carolina Neurosurgery and Spine Associates; ${ }^{2}$ Department of Neurological Surgery, Carolinas Medical Center, Charlotte, North Carolina; ${ }^{3}$ Department of Neurological Surgery, Vanderbilt University Medical Center, Nashville, Tennessee; ${ }^{4}$ Department of Orthopaedics, University of Louisville School of Medicine, Louisville, Kentucky; ${ }^{5}$ Heart Health Strategies, Washington, DC; ${ }^{6}$ Department of Neurological Surgery, School of Medicine and Public Health, University of Wisconsin, Madison, Wisconsin; and ${ }^{7}$ Columbia University College of Physicians and Surgeons, New York, New York

\begin{abstract}
Clinical registries have emerged in the current resource-restricted environment of modern medicine as useful and logical mechanisms for providing health care stakeholders with high-quality data related to the safety, effectiveness, and value of specific interventions. Temporal and qualitative requirements for data acquisition in the context of clinical registries have rapidly expanded as clinicians and other stakeholders increasingly recognize the central importance of this information to the intelligent transformation of health care processes. Despite the potential of more robust clinical data collection efforts to advance the science of care, certain aspects of these newer systems, particularly the prospective, longitudinal acquisition of clinical data and direct patient contact, represent areas of structural overlap between emerging quality improvement efforts and traditional models of human subjects research. This overlap has profound implications for the design and implementation of modern clinical registries. In this paper, the authors describe the evolution of clinical registries as important tools for advancing the science of practice, and review the existing federal regulations that apply to these systems.
\end{abstract}

(http://thejns.org/doi/abs/10.3171/2012.10.FOCUS12300)

KEY WORDS $\bullet \quad$ Office for Human Research Protections $\bullet$ Office for Civil Rights
Health Insurance Portability and Accountability Act $\bullet$ human subjects research
clinical registry $\bullet \quad$ patient-reported outcome $\bullet$ privacy $\bullet$
National Neurosurgery Quality and Outcomes Database

\footnotetext{
Abbreviations used in this paper: AANS = American Association of Neurological Surgeons; CFR = Code of Federal Regulations; $\mathrm{CNS}=$ Congress of Neurological Surgeons; HHS = US Department of Health and Human Services; HIPAA = Health Insurance Portability and Accountability Act; IRB = Institutional Review Board; $\mathrm{N}^{2} \mathrm{QOD}=$ National Neurosurgery Quality and Outcomes Database; NPA = NeuroPoint Alliance, Inc.; NSQIP = National Surgical Quality Improvement Project; OCR = Office for Civil Rights; OHRP = Office for Human Research Protections; PHI = protected health information; VIMPH = Vanderbilt Institute for Medicine and Public Health.

* This paper was based heavily on and incorporates text from the document "The National Neurosurgery Quality and Outcomes Database ( $\left.\mathrm{N}^{2} \mathrm{QOD}\right)$ : A Prospective Registry for Quality Reporting (v5)" and the multisociety memorandum "ANPRM on Human Subjects Research Protections: Enhancing Protections for Research Subjects and Reducing Burden, Delay, and Ambiguity for Investigators (October 2011)." Permission for liberal reuse of the material was given by NPA and the AANS, respectively. The primary author of this paper authored the copyrighted document and was a major contributor to the memorandum.
}

$\mathrm{T}$ THE rapid rise of health care costs, coupled with increasing societal demands for greater physician accountability, has dramatically shifted the focus of all stakeholders toward critical analyses of quality and cost in health care delivery. ${ }^{8}$ In this environment, clinical data registries have emerged as useful and logical mechanisms for providing stakeholders with high-quality data related to the safety, effectiveness, and value of specific interventions. In this analysis, we describe the evolution of clinical registries to comprehensive tools for evaluating the quality of care, review the existing federal regulations that apply to these systems (using the National Neurosurgery Quality and Outcomes Database [N²QD] experience as a case study), and discuss potential methods to adequately protect the security and well-being of patients while avoiding unnecessary administrative burdens to clinical quality improvement efforts.

\section{The Evolution of Clinical Registries}

Currently, two national surgical quality registries ex- 
ist that have demonstrated value and validity in reporting and improving the quality of surgical care. The American College of Surgeons National Surgical Quality Improvement Project (NSQIP; http://www.facs.org/cqi/outcomes. html) and The Society of Thoracic Surgeons National Database (http://www.sts.org/quality-research-patient-safety /sts-public-reporting-online/sts-national-database-today) are successful platforms for quality reporting and riskadjusted performance measurement that have been widely used by both hospitals and surgeon practice groups to define and promote best practice standards.

The Society of Thoracic Surgeons and NSQIP registries represent important advances in the emerging quality care paradigm. Their reliance on retrospective acquisition of clinical information from the existing medical record and a focus on short-term clinical outcomes has obviated the need for direct patient contact and has generally simplified the management of these pioneering projects, thus facilitating their widespread and practical implementation. However, continued gaps in the evidence supporting clinical practice parameters, as well as a growing emphasis on patient-centered and value-based payment approaches to care in both the public and private sector, highlight the emerging importance of prospectively captured longitudinal data and the incorporation of more robust data elements such as patient-reported outcomes. ${ }^{1,3,5}$

Both longitudinal data and patient-reported outcomes are fundamental to the comprehensive assessment of the quality and appropriateness of specific clinical interventions, particularly those typically encountered in neurosurgical practice. The former makes it possible to determine the sustainability of treatment effects, while the latter permits a more comprehensive picture of the quality of care received. Blue Cross Blue Shield's Blue Distinction Program (http://www.bcbs.com/why-bcbs/bluedistinction/blue-distinction-program-overv.html), which requires the collection of long-term clinical outcomes data to establish that a facility meets selected criteria thresholds, is an example of emerging programs designed to track the extended results and cost effectiveness of clinical care. Newly established surgical registries, such as the $\mathrm{N}^{2} \mathrm{QOD}$ and the American Joint Replacement Registry, now incorporate both longitudinal and patient-focused components in their design. Temporal and qualitative requirements for clinical data acquisition have therefore rapidly expanded as clinicians and other stakeholders increasingly recognize the central importance of this information to the intelligent transformation of health care processes.

Despite the potential of more robust clinical data collection efforts to advance the science of care and more completely inform health care decision making and resource utilization, these newer systems are associated with a variety of challenges including, but not limited to, the costs of registry development and execution, applicability of systems to a wide spectrum of practice settings, relevance of data variables to major stakeholders, data reliability/validity, proper data use and ownership, and increased regulatory burdens. With respect to the last issue, the need for prospective, longitudinal acquisition of clinical data and direct patient contact represents an area of structural overlap between emerging quality improvement efforts and traditional models of human subjects research. This overlap has profound implications for the design and implementation of modern clinical registries.

\section{Comprehensive Registry Designs: Implications of the Relationship to Human Subjects Research}

It is now clear to many health care stakeholders that the growth of practice science, particularly efforts to collect, analyze, and apply data derived from daily patient care, has greatly outpaced the development and revision of regulations designed to protect human subjects and ensure the security of patient information. As acknowledged by the US Department of Health and Human Services (HHS) in its recent proposal to potentially enhance federal regulations overseeing research on human subjects and reduce unnecessary regulatory burdens and ambiguity, ${ }^{4}$ human subject protections need to be modernized and simplified to meet a rapidly evolving data collection enterprise that was not envisioned when these federal regulations were first developed. The steady implementation of public- and private-payer patient safety initiatives and quality reporting mandates, an increased emphasis on value of care and comparative effectiveness research, and the widely expressed need for longitudinal and patient-reported outcomes data necessitate enlightened regulatory oversight.

Because the present standards surrounding human subjects research and patient privacy (governed by the "Common" and "Privacy" Rules, respectively) are more developed and specific than those for quality improvement, the latter efforts are often subject to research standards established specifically for the former. ${ }^{6}$ This is particularly the case when prospective data collection and ongoing patient contact, often characteristics of human subjects research, become components of quality improvement efforts. This situation is complicated by a fundamental lack of consistency in local interpretations of Common and Privacy Rules provisions relevant to clinical registries. As such, if IRBs are unsure of the relationship between federal guidelines and quality efforts, there is often a bias toward classifying certain quality projects as human subjects research.

A research designation has substantial implications for quality improvement efforts. The requirement for formal IRB oversight can significantly increase the administrative burdens and costs of clinical data collection. Most importantly, as clinical registries rely on serial evaluation of patient outcomes, the requirement for informed consent, in particular, can undermine the quality and consistency of collected data and compromise the validity of data assessments. Several groups have now pointed out the extent to which traditional research requirements are a significant hindrance to quality improvement efforts. Armstrong et al. ${ }^{2}$ offered a dramatic example of how the requirement for informed consent can significantly decrease the number of patients available for quality outcomes research and introduce selection bias in data collection for patient registries. The study, which analyzed the University of Michigan 


\section{Regulatory considerations for prospective patient care registries}

Acute Coronary Syndrome Registry prior to and after the implementation of the Health Insurance Portability and Accountability Act of 1996 (Public Law 104-191), showed that participation rates decreased from $96 \%$ before HIPAA to $34 \%$ after HIPAA. Additionally, patients who gave consent were not representative of the larger treatment group, and were more likely to be older, married, and white, and exhibited lower mortality rates at 6 months. Simply put, when a requirement for informed consent exists, patients who are willing to give consent often comprise a nonrepresentative subset of the population of interest.

\section{The N²QOD: Brief Outline and Early Regulatory Experience}

The experience of the $\mathrm{N}^{2} \mathrm{QOD}^{7}$ further illustrates ways that the current regulatory framework poses challenges to newer forms of clinical data collection, particularly those related to quality improvement.

The $\mathrm{N}^{2} \mathrm{QOD}$ is designed to serve as a continuous national clinical registry for neurosurgery along the lines of the very successful Society of Thoracic Surgeons National Database. The primary purpose of this registry is to track the quality of surgical care for the most common neurosurgical procedures, as well as provide practice groups and hospitals with immediate infrastructure for analyzing and reporting the quality of their neurosurgical care, including risk-adjusted benchmarks. Like other emerging and more comprehensive clinical data collection systems (such as the American Joint Replacement Registry [http://orthodoc.aaos.org/ajrr/grp_about.cfm]), $\mathrm{N}^{2} \mathrm{QOD}$ promotes quality assurance through the collection of patient-centered, nonadministrative data.

To better support the administration of this evolving effort, the American Association of Neurological Surgeons (AANS) - in cooperation with a broad coalition of other neurosurgical societies including the Congress of Neurological Surgeons (CNS), the Society of Neurological Surgeons, and the American Board of Neurological Surgery-formed the NeuroPoint Alliance (NPA), an independent nonprofit entity that will provide neurosurgeons with an internet-based data management platform for collecting, evaluating, and improving upon neurosurgical outcomes. The NPA has partnered with the Vanderbilt Institute for Medicine and Public Health (VIMPH) to manage the collection and analysis of standardized data across neurosurgical practices.

Information flow in the $\mathrm{N}^{2} \mathrm{QOD}$ is similar to that used in other clinical registries. Participating $\mathrm{N}^{2} \mathrm{QOD}$ sites collect data related to the routine care of patients undergoing specified types of neurosurgical procedures at their facilities each week. Those data are entered into a HIPAA-secure portal and transmitted to VIMPH for analysis. Quality scientists (scientists who study quality) use de-identified forms of these data to establish and refine national benchmarks for the quality of neurosurgical procedures and also develop risk-adjusted quality reports that are then transmitted to the practice sites. It is acknowledged that de-identified aggregate registry data could potentially be used by researchers ("secondary analysis" researchers, see below) in as yet undefined future research efforts designed to advance the science of clinical care.

Data collection in the $\mathrm{N}^{2} \mathrm{QOD}$ requires direct patient contact, primarily to obtain information not available in the standard patient record such as data from patientreported outcome instruments, and also the retention of identifying links to individual patients to facilitate longitudinal follow-up and maximize the analytical value of the quality data collected, such as by linking to other data sets to determine cost, outcome, and value of care. Importantly, this effort is not primarily designed to produce "generalizable knowledge" (see below).

The NPA regards the systematic collection of outcomes data that are primarily intended to promote quality improvement-including the collection of patient-reported outcomes through validated assessment instrumentsas an important extension of existing methods of neurosurgical care. As such, and based on its interpretation of existing federal guidelines relevant to clinical registries, our organization believes that the $\mathrm{N}^{2} \mathrm{QOD}$ project is best designated as a nonresearch, clinical quality improvement effort, exempt from IRB review and the requirement for informed consent. Despite this position, our organization believed it was most appropriate to allow individual IRBs to evaluate the $\mathrm{N}^{2} \mathrm{QOD}$ Project Description prior to its local implementation.

Project descriptions were originally forwarded to multiple practice sites in the fall of 2010. By February 2011, IRBs at 23 institutions from around the country representing both community and academic practices had completed their review of the $\mathrm{N}^{2} \mathrm{QOD}$ project. Eighteen of the 23 sites classified the $\mathrm{N}^{2} \mathrm{QOD}$ as quality improvement and waived the requirement for IRB review. Interestingly (and disturbingly), the remaining 5 IRBs classified the same project description as research, and insisted on full IRB oversight and the requirement for informed consent.

In reviewing the local justifications for research designations, the following patterns emerged:

1) The project was generally reviewed through the lens of the so-called "Common Rule," which is a set of regulations used by numerous federal agencies to regulate human subjects research. ${ }^{9}$

a. Direct patient contact, longitudinal design, the gathering of data that was deemed to not be part of the "standard of care" (i.e., patient-reported outcomes), and the possibility of subsequent use of the data for research purposes were most commonly listed as reasons to apply research standards to the registry.

2) The project was also reviewed with respect to the HIPAA "Privacy and Security Rules" which are federal regulations used to address security and privacy considerations regarding individually identifiable patient information (defined by HIPAA as protected health information or PHI). ${ }^{10,11}$

a. Any transfer of PHI was sometimes cited as a reason to mandate the application of research standards to the registry.

After reviewing the initial institutional responses to the $\mathrm{N}^{2} \mathrm{QOD}$ Project Description, the NPA came to the following conclusions:

1) Several centers seemed to be either misinterpreting or inappropriately applying elements of the Common Rule to this quality improvement effort. Furthermore, the justifications for 
assigning research status seemed to vary significantly from center to center, which was likely related to ambiguous or irrelevant wording in the existing regulations.

2) The Privacy and Security Rules provide significantly greater protections for the safeguarding of patient data in this quality effort, which we believed to be representative of other emerging national quality programs. Despite this, some sites appeared to be unaware of important exceptions to HIPAA requirements for patient authorization relevant to quality improvement efforts.

\section{Addressing Unnecessary Regulatory Burdens to Quality Efforts}

Our analysis of this situation suggested that the current regulatory structure fails to recognize that data collection for quality improvement purposes poses no direct physical risk to individual patients. The only risk that may exist is informational, such as unauthorized release or other inappropriate use of personal data. However, and as HHS pointed out in its recent Advance Notice of Proposed Rulemaking, HIPAA's Privacy Rule already addresses many of these informational risks by imposing restrictions on how certain identifiable health information collected by health plans, health care clearinghouses, and health care providers may be used and disclosed. In addition, the HIPAA Security Rule requires that these entities implement certain administrative, physical, and technical safeguards to protect this information when in electronic form from unauthorized use or disclosure. As such, it seems extraneous to hold registries to Common Rule consent requirements if they already comply with HIPAA patient protections.

Although the NPA, like most health care stakeholders, has embraced the intention of reforms and efforts designed to improve the quality and value of care, our organization determined that variable regional interpretation and application of current federal regulations (particularly the Privacy and Common Rules) has created significant impediments to accomplishing these goals. A clear need exists for regulatory agencies to establish updated, appropriate standards for quality improvement activities that will adequately protect patients, but not unnecessarily burden quality improvement efforts. In the absence of such guidance, it will be difficult or impossible for clinicians to participate in the full spectrum of quality efforts now being mandated by regulatory bodies in the public and private health care arenas.

Significant revisions to existing federal regulations are likely to take years to develop. In the short term, the AANS/CNS, acting on behalf of the NPA and organized neurosurgery, chose to seek clarification of the intent of existing regulations with respect to registries designed to promote quality improvement. Specifically, our organization sought guidance from the HHS OHRP regarding the application of existing federal regulations (in particular the Common Rule) to the $\mathrm{N}^{2} \mathrm{QOD}$ project. We subsequently had communications with the HHS OCR, which is responsible for oversight of the Privacy Rule. Our communications with these federal agencies produced information of likely value to all existing and developing clinical registry efforts. A summary of the OHRP and OCR instructions, along with a brief review of the relevant federal regulations, is offered in the following sections.

\section{HIPAA, the Common Rule, and N²QOD}

The HHS, in response to mandates authorized under HIPAA, set forth the Privacy and Security Rules (45 CFR Part 160) and the Common Rule (Subpart A of 45 CFR Part 46). These regulatory rules guide the practice of all federally funded agencies and most private agencies with regard to oversight of ethical research standards; the security, privacy, and transfer of health data; and the use of PHI. Note that although the Common Rule only regulates federally funded or supported research, many academic medical centers apply the Common Rule to all research in the interest of ensuring that all research is performed according to the highest ethical standards. Adherence to Common Rule standards is also often required by federal-wide assurance agreements between institutions and the federal government.

The Common Rule applies to research involving human subjects. These terms are defined in the regulations as follows:

1) Research: a systemic investigation, including research development, designed to develop or contribute to generalizable knowledge (which is generally interpreted to refer to knowledge that could be applied to populations outside of the population under analysis).

2) Human subject: a living individual about whom an investigator conducting research obtains

a. Data through interaction with the individual, or

b. Identifiable private information

Federally funded research involving human subjects is subject to the jurisdiction of the Common Rule, requiring IRB review and informed consent or waiver of consent. The Common Rule makes no distinction between the primary and secondary intents of data collection. As mentioned above, some institutions choose to apply these regulations to all forms of human research. However, the regulations contain qualifying, but sometimes confusing, language regarding the definitions of research and human subjects that impacts the applicability of various federal regulations to quality improvement efforts. Important clarifications are discussed below.

The Privacy Rule also defines important terms that are relevant to the determination of the research status of clinical data collection projects. These regulations also define situations in which such data can be obtained without obtaining expressed authorization from individual patients:

1) Protected Health Information (PHI): Under HIPAA, PHI is individually identifiable health information that requires patient authorization (or a waiver of authorization, often obtained from a local IRB) for disclosure unless an exemption applies.

a. HIPAA applies to "covered entities" (i.e., providers, health plans) and business associates (entities that provide services for the covered entity).

An important exemption to the requirement for patient authorization relates to activities characterized as "health care operations." Relevant to this discussion, 


\section{Regulatory considerations for prospective patient care registries}

health care operations include quality assurance and improvement projects. In these settings, covered entities can share data with business associates if they enter into business associate agreements with the business associate; a full explanation of business associate agreements can be found at the HHS OCR website (http://www.hhs.gov/ocr/ privacy/hipaa/understanding/summary/index.html).

Unlike the Common Rule, the Privacy Rule distinguishes between research and quality improvement projects based on whether the primary purpose of the analysis is to promote quality improvement. Specifically, the regulations state that patient data can be used to summarize health care provider performance or be put to any other purpose that meets the definition of health care operations under the Privacy Rule; this pertains to conducting quality assessment and improvement activities, including outcomes evaluation and development of clinical guidelines, provided that obtaining generalizable knowledge (research) is not the primary purpose of any studies resulting from such activities (45 CFR Part 164.501).

The $\mathrm{N}^{2} \mathrm{QOD}$ and its network of practicing neurosurgeons and hospitals, like similar clinical registries, is not primarily intended to serve as a research initiative. The primary purpose of participation in $\mathrm{N}^{2} \mathrm{QOD}$ is for health care operations, defined by HIPAA to include quality assessment and improvement activities, including outcomes evaluation. Any future use of the aggregate database for as yet undefined research activities would be governed by specific provisions outlined in 45 CFR (see following sections).

Per these regulations, IRB review of registry efforts is not required if clinical data collection is used for purposes of health care operations such as clinical care, administrative use, or quality assessment. Furthermore, all $\mathrm{N}^{2} \mathrm{QOD}$ participating sites fall under the description of "covered entities" per 45 CFR Part 160. As such, PHI can be disclosed by registry sites to an entity that performs functions or services on behalf of the covered entity (the business associate, in this case, VIMPH) as long as the practice sites have entered into a business associate agreement with the recipient of the data. The business associate agreement between $\mathrm{N}^{2} \mathrm{QOD}$ sites and the NPA contains provisions for the appropriate safeguarding of PHI.

\section{Support for the Designation of "Nonregulated, Quality Improvement Project": OHRP Opinions}

The AANS/CNS approached the HHS in May 2011 with a request to review the $\mathrm{N}^{2} \mathrm{QOD}$ (http://www.neuro point.org/pdf/N2QOD\%20Project\%20Desc\%20Attach ments-Regulatory\%20Documents_March2012.pdf). On August 11, 2011, the AANS/CNS received a preliminary written communication from OHRP, which also included comments on theoretical regulatory considerations that we posed as part of our outreach. This document was discussed in detail at a multistakeholder meeting at the White House Executive Office Building on August 25, 2011. In attendance were representatives of the Office of the President, the Centers for Medicare and Medicaid Services, the FDA, the US Department of Veterans Affairs, the OHRP, the OCR, and a consortium of registry design- ers including the NPA, the North American Spine Society, the Academy of Orthopedic Surgeons, the American College of Surgeons, and the American Cardiology Society. Subsequent to this conference, the AANS/CNS engaged in additional dialogue with OHRP and eventually sent a detailed communication, summarizing the multiple areas in which we were seeking guidance relevant to the $\mathrm{N}^{2} \mathrm{QOD}$ project (http://www.neuropoint.org/pdf/N2QOD $\% 20$ Project\%20Desc\%20Attachments-Regulatory\%20 Documents_March2012.pdf). On December 29, 2011, the AANS/CNS received final guidance from the OHRP regarding our outreach (http://www.neuropoint.org/pdf/ N2QOD\%20Project\%20Desc\%20Attachments-Regula tory\%20Documents_March2012.pdf). A summary of the OHRP conclusions was as follows:

1) First, OHRP would not consider the $N^{2} Q O D$ practice groups (registry sites) to be institutions that are engaged in human subjects research in that the practice groups are only releasing private identifiable information obtained through the administration of standard clinical care to patients (see Scenario B (6) in the OHRP guidance document titled "Guidance on Engagement of Institutions in Human subjects Research," available on the OHRP website at http://www.hhs.gov/ohrp/policy/ engage08.html.).

a. The Human Subjects Research regulations (45 CFR 46, The Common Rule) do not apply to the releasing activity of the groups regardless of the funding status of the clinical registry project and independent of the potential use of private health information for research purposes.

2) Second, the $N^{2} Q O D$ registry is not supported or regulated by HHS or any other federal agency, and the registry institution (VIMPH) has not elected to extend the application of the regulations to non-federally funded research activities via the HHS Federalwide Assurance. Consequently, this registry institution does not have to meet any of the requirements of the regulations, including review by an IRB, for this non-HHS funded activity.

a. Interestingly, OHRP does regard the development of national practice benchmarks by VIMPH to be "human subjects research" as defined by the regulations since these benchmarks are considered to be "generalizable knowledge." However, the Common Rule does not apply in the absence of federal funding for or regulation of the project, and IRB review and informed consent are not required. Should federal funding someday support this project, IRB review would be required, but this could be obtained from a single IRB. Under these circumstances, it is highly likely that the registry institution would apply for an IRB waiver of informed consent.

b. The reporting of analyzed, aggregate, de-identified data back to practice sites by the registry institution is not human subjects research since de-identified patient data are not regulated by the Common Rule (see following).

3) Third, OHRP would consider the activity of the institutions of the secondary analysis researchers (i.e., those using de-identified data for later research) as not being research involving "human subjects," according to the definition of "human subject" in the regulations, which is "... a living individual about whom an investigator (whether professional or student) conducting research obtains (1) Data through intervention or interaction with the individual, or (2) Identifiable private information...." (45 CFR Part 46.102(f)). Since the secondary analysis researchers are obtaining de-identified data, and are not intervening or interacting with the subjects in any way, the regulations do not apply to the activities of these secondary 
analysis researchers, and there is no requirement for review and approval by an IRB.

4) Fourth, (where appropriate) OHRP supports the use of single or central IRB review and approval of research conducted by clinical registries in circumstances in which more than one institution is engaged in research.

In its communication, the OHRP reaffirmed that it is important to "provide appropriate safeguards for the protections of the rights and welfare of human subjects." It also, however, recognized that "serious questions and concerns have been raised about the appropriateness of oversight of quality improvement and research activities in health care" and stated that it "share[s] (organized neurosurgery's) concerns about creating unnecessary impediments to improving the quality of health care or to the use of data to improve health care."

\section{Clarification of Clinical Data Transfer and Use Regulations: OCR Opinions}

The AANS/CNS also approached the OCR with a request to review and provide written guidance regarding provisions in the Privacy and Security Rules relevant to the $\mathrm{N}^{2} \mathrm{QOD}$ program. We were informed that this agency does not routinely provide such written guidance. The HHS OCR representatives did, however, provide verbal guidance at the White House Conference and in subsequent communications (phone conferences on March 15, 2012, and September 20, 2012; e-mails on March 15, 2012) that included references to specific information on the OCR and NIH websites. OCR's primary guidance regarding the $\mathrm{N}^{2} \mathrm{QOD}$ and similar registry projects is summarized below.

The authors refer those interested to HHS guidance regarding the Privacy Rule and research/health care quality improvement, which is posted online at the following addresses:

http://www.hhs.gov/ocr/privacy/hipaa/faq/health_ information_technology/544.html

http://privacyruleandresearch.nih.gov/healthser vicesprivacy.asp

Relevant OCR guidance with respect to registry projects includes the following:

1) HIPAA allows for disclosure of PHI without patient authorization under the exemption for health care operations (which includes quality assurance projects).

2) OCR confirms that registries may serve as a business associate for multiple covered entities/sites and collect and analyze PHI from those covered entities for quality improvement purposes as long as:

a. A HIPAA-compliant business associate agreement is in place between the registry and each of the sites.

b. The data given back to the sites is de-identified and in aggregate form.

3) The Privacy Rule permits covered entities to use and disclose data that have been de-identified without obtaining an Authorization and without further restrictions on use or disclosure because de-identified data are not PHI, and therefore are not subject to the Privacy Rule.

a. Note however: the process of de-identifying PHI constitutes a use of PHI. Thus, a business associate may only de-identify PHI it has on behalf of a covered entity to the extent that the business associate agreement authorizes the business associate to do so (such an authorization is present in the $\mathrm{N}^{2} \mathrm{QOD}$ business associate agreement). However, once PHI is de-identified in accordance with the HIPAA Privacy Rule, it is no longer PHI and, thus, may be used and disclosed by the covered entity or business associate for any purpose.

i. It follows, therefore, that secondary (i.e., retrospective) use of de-identified data for research purposes is not an activity regulated by the privacy rule.

4) The Privacy Rule distinguishes between research and studies for quality assessment and improvement purposes based on whether the primary purpose of the study in question is to obtain generalizable knowledge. Although generalizable knowledge in the form of national benchmarks for performance will be generated in the $\mathrm{N}^{2} \mathrm{QOD}$ project, the primary purpose of the project is to promote health care quality improvement.

a. In situations in which the primary purpose of the study is for health care operations, the covered entity may use or disclose PHI for the study without patient authorization.

b. If PHI was being submitted primarily for research purposes, then the use or disclosure of PHI for such study would have to be made in accordance with the Privacy Rule's provisions for the use and disclosure of PHI for research. For example, an IRB or a Privacy Board may waive or alter the Authorization requirement, as long as certain criteria at section 164.512(i)(2)(ii) are met (i.e., the use or disclosure of PHI involves no more than minimal risk to the privacy of individuals and the research could not practicably be conducted without the requested waiver or alteration or without access to and use of the PHI conditions which exist in the $\mathrm{N}^{2} \mathrm{QOD}$ registry-see below). The OCR has clearly stated that such a waiver of authorization could be obtained from a single, or central, unaffiliated IRB or Privacy Board.

\section{Summary of OHRP and OCR Opinions}

In summary, the $\mathrm{N}^{2} \mathrm{QOD}$ (and, by extension, similar registries) regulatory obligations with respect to the Common and Privacy Rules are as follows:

\section{1) Practice Sites}

a. Common Rule: according to OHRP does not apply to practice sites submitting data to $\mathrm{N}^{2} \mathrm{QOD}$ as part of the normal clinical care of their patients, regardless of the funding status of the project.

b. Privacy Rule does apply

i. The business associate agreement must specify permission to de-identify data.

ii. Data must be handled in a HIPAA-compliant fashion.

iii. If PHI is used for research (as opposed to de-identified data), a waiver of HIPAA authorization (generally obtained by the business associate) is required. A single-institution waiver is sufficient.

2) The Registry Institution

a. Common Rule: does not apply to present (non-federally funded) activities.

i. Single institution "waiver of informed consent" will be required should federal funds be used to support the project.

b. Privacy Rule: does apply 


\section{Regulatory considerations for prospective patient care registries}

\author{
i. The business associate agreement must specify per- \\ mission to de-identify data.
}

ii. If PHI is used for research, a single/central waiver of HIPAA authorization is required.

It is important to note that the neither the Privacy nor Common Rules apply to the analysis and/or transfer of de-identified data sets, that is, information that contains no personal identifiers or unique identifying numbers, characteristics, or codes. The primary significance of this lack of regulatory jurisdiction is that IRB review and patient consent/authorization for research involving such data are not required.

\section{Provisions for Possible Alternative Interpretations of Federal Guidelines: Rationale for Requesting Waiver of Consent}

In the rare circumstance that a local IRB may designate this project as a research effort despite the OHRP opinion that the participation of sites in the $\mathrm{N}^{2} \mathrm{QOD}$ project does not constitute "human subjects research," the NPA recommends that sites modify the project description into a clinical protocol submission based on their individual IRB's document templates. Furthermore, in these instances we suggest that a "Waiver of Consent" be requested. The rationale for requesting such a waiver is provided below.

All variables included in the $\mathrm{N}^{2} \mathrm{QOD}$ registry are recorded into the medical record as accepted best practice of perioperative spine care. In many centers, assessment of pain, disability, and health status using the Back and Leg Pain Scale, Oswestry Disability Index, and EQ-5D are performed as a part of standard of care. Although not all centers currently collect the data points included in these instruments on a regular basis, all centers participating in $\mathrm{N}^{2} \mathrm{QOD}$ have decided to do so as part of their routine care because it is considered a best practice in both neurosurgery and orthopedic spine surgery. The extent of pain (visual analog scale), the degree to which pain affects patients' physical function (Oswestry Disability Index), and patients' general health state (EQ-5D) are routinely a part of preoperative assessment of the need for spine surgery, as well as postoperative assessment of response to such surgery. Furthermore, no interventions or alterations in direct patient care will occur with participation in $\mathrm{N}^{2} \mathrm{QOD}$, which is entirely noninterventional and observational. All treatment, management, and follow-up decisions are determined solely by the surgeon and the patient.

With assessment of pain, disability, and state of health, along with basic demographic, comorbidity, and clinical variables being acquired as accepted best practice (which are standard of care at most institutions), measurements or interventions outside of the routine standard of care will not be required for the $\mathrm{N}^{2} \mathrm{QOD}$ quality improvement initiative.

The criteria met for waiver-of-consent exemption per the Common Rule and the Privacy Rule are listed below: ${ }^{7}$

Common Rule Criteria

1. Quality assessment involves minimal to no risk to the subjects: The only known risk to patients is the possible loss of confidentiality, which has been guarded against as per the data protection protocol specified in the $\mathrm{N}^{2} \mathrm{QOD}$ Project Description. Direct patient identifying information is transmitted via secure web certificate and risk of confidentiality is minimal.

2 . The waiver will not adversely affect the rights and welfare of the subjects: The $\mathrm{N}^{2} \mathrm{QOD}$ is non-interventional, does not affect the subject's rights for patient care, and does not interfere with his/her welfare. In fact, clinical registries do just the opposite. Using registry data to establish local or national quality benchmarks, for example, can actually benefit patients by ensuring more accurate diagnoses, more appropriate treatments and procedures, and better outcomes overall. Furthermore, the acquisition of long-term patient-reported outcome data are now becoming the standard of care in multiple disease states and (as mentioned in previous sections) will soon be mandated by public and private payers.

3 . The research could not practicably be performed without the waiver or alteration: The $\mathrm{N}^{2} \mathrm{QOD}$ is implemented for quality reporting and benchmarking outcomes and not research. The requirement for traditional informed consent would significantly impede the ability of clinicians to review serially treated patients, thus introducing unacceptable bias into any subsequent analysis and undermining the validity of any reported data.

4. Whenever appropriate, the subjects will be provided with additional pertinent information after participation: The $\mathrm{N}^{2} \mathrm{QOD}$ registry is non-interventional and thus providing information to patients is not applicable. Other than information that providers participate in quality reporting initiatives, no additional information about participation will be provided.

Privacy Rule Criteria

1. The use or disclosure of the PHI involves no more than minimal risk: a) There is an adequate plan to protect health information identifiers from improper use and disclosure (per protocol on data protection); b) Since $N^{2}$ QOD may use quality reporting data for quality improvement, maintenance of patient identifiers is required. All data will be collected under a Business Associate agreement between the Covered Entity and the NPA. Any subsequent use of data for analyses of the participants will be performed using the minimal necessary data to accomplish the purpose and under an agreement, which passes down the required obligations of the Business Associate and Data Use agreements. For this reason, the identifiers will not be destroyed; c) There are written assurances that the PHI will not be reused or disclosed to any other entity, except as required by law.

2. The research could not practicably be conducted without the waiver or alteration: see Common Rule criterion 3.

\section{Emerging National Standards for Surgical Outcomes Registries}

The successful NSQIP and Society of Thoracic Surgeons registries, both of which have been uniformly recognized as quality improvement initiatives, have generally been granted waiver of consent or outright IRB review exemption by participating institutions. The operational protocol, handling of data, and use of data for neurosurgery's $\mathrm{N}^{2} \mathrm{QOD}$ mimics that of both general surgery's NSQIP and cardiothoracic surgery's Society of Thoracic Surgeons National Database. The Society of Thoracic Surgeons has, within the last few years, included PHI in the collected variables to improve the value of the registry by allowing linkage with long-term data sets. The major difference be- 
tween neurosurgery's effort and the other surgical registries is the inclusion of long-term patient-reported outcomes in the $\mathrm{N}^{2} \mathrm{QOD}$ data set. As mentioned previously, the acquisition of such data is essential for the meaningful evaluation of surgical efficacy related to many of the disorders that neurosurgeons treat. Furthermore, such information will soon be required by a variety of payers and its acquisition will be mandated by federal regulations.

Other publications have described the rationale for exemptions made by IRB committees, waiver of consent, and use of PHI in the Society of Thoracic Surgeons National Database and other national quality registries (http://www.facs.org/cqi/outcomes.html). ${ }^{4-6}$

\section{Potential Use of Registry Data for Research}

Although clinical registries such as the $\mathrm{N}^{2} \mathrm{QOD}$ are primarily intended as prospective quality improvement tools, the significant potential of such databases to improve quality of care through retrospective analysis of data subgroups makes it probable that clinician scientists will request to analyze this data set for research purposes in the future. Rules and mechanisms to guide this potential use of data are now being developed by the NPA. We refer those interested to the excellent analysis provided by Dokholyan et al. ${ }^{6}$ regarding this situation. In summary, we believe that any future use of identified $N^{2}$ QOD data sets for research purposes would require IRB oversight. As noted above, we have received specific guidance from OHRP that secondary analysis of de-identified data does not constitute "human subjects research" and therefore is not subject to the jurisdiction of 45 CFR Part 46. Furthermore, should such research be undertaken, a single waiver of patient authorization will be obtained from the institution conducting the analysis (likely VIMPH) to satisfy relevant provisions on the Privacy Rule (as explained above).

\section{Impact of Federal Guidance on Local Review of the $\mathrm{N}^{2} \mathrm{QOD}$}

The impact of federal guidance on local IRB review of the $\mathrm{N}^{2} \mathrm{QOD}$ project has been significant. Copies of all written federal instructions, along with summaries of relevant federal guidelines and verbal communications with OCR, were circulated to participating centers in the spring of 2012. As of September 2012, 37 IRBs have formally reviewed (or re-reviewed) the project description with many other sites in various stages of institutional review. Presently, 36 of $37 \mathrm{~N}^{2} \mathrm{QOD}$ sites have classified neurosurgery's project as quality improvement and have waived the requirement for IRB review. A single registry site continues to designate this project as "research," but has waived the requirement for informed consent. This institution is currently reevaluating the project based on the OHRP's recent written opinion.

Major sites that have received IRB determinations of "not regulated" quality improvement status for the $\mathrm{N}^{2} \mathrm{QOD}$ project include Vanderbilt University; University of Illinois; University of Alabama; University of California, San Francisco; University of California, Los Ange- les; University of Virginia; Cornell/NewYork-Presbyterian; University of Michigan; Semmes-Murphey Clinic; Barrow Neurological Institute; Carolinas Healthcare System; University of Utah; and the University of Louisville. We have received communications from other registry projects that the information gained from this federal outreach has greatly facilitated IRB interactions and the implementation of registry-specific processes.

\section{Future Directions}

While the clarifications received to date from federal agencies such as the OHRP and OCR have been extremely helpful, they were in response to a single, focused inquiry and have not yet been widely publicized. There is also remaining confusion as to why using collected data to establish local or national benchmarks for quality improvement purposes would trigger the application of these regulations.

A multisociety coalition has requested that both the OHRP and OCR issue formal statements that outline the aforementioned interpretations in clear and precise terms that are easily understandable to clinicians, health care administrators, IRB members, attorneys, and even patients (http://sts.org/sites/default/files/documents/pdf/news letters/Beltway/HHS-Proposed-Rulemaking-HumanSubjects-Research-Protections.pdf). These guidance statements (or at least links to them) should be prominently displayed on the OHRP and OCR websites, as well as any other relevant federal government websites frequented by the public. These statements should clarify the following points specifically:

1. A hospital, medical group, or individual clinician that submits data to a registry based solely on clinical care encounters with patients and is not involved in research itself, would not be engaged in human subject research and therefore would not be subject to the Common Rule's patient consent requirements.

2. If a registry is engaged in activities that meet the Common Rule's definition of human subjects research, it would only need a single IRB waiver of patient authorization, on which all participating sites could rely.

3 . That registry reporting and data collection for quality improvement purposes is considered part of "health care operations" as defined under HIPAA's Privacy Rule and therefore should not require individual authorization or consent, or otherwise subject covered entities to duplicative regulatory requirements.

While immediate clarification of existing regulations is critical to ensure existing registries can successfully collect data at a level needed to drive improvements in patient care, we have also requested that the federal government consider these more fundamental changes to clinical research regulations over the long term:

1. Because HIPAA Privacy and Security Rules provide the same or greater protection for patient data as the Common Rule, there is no need to apply the Common Rule for data collection activities where HIPAA compliant policies, procedures, and waivers are already in place. Therefore, we have requested that OHRP issue guidance that the Common Rule does not apply to the collection of identifiable patient information where the entity collecting the data (such as a clinical data registry) is 


\section{Regulatory considerations for prospective patient care registries}

engaged in standard patient care and is in compliance with all applicable HIPAA requirements.

2. The OHRP should also amend its definition of human subject research so that it takes into account the extent to which a patient is at risk for physical harm. It should not be based on whether individually identifiable patient data were exchanged (informational risks) because there are already protections in place for these types of risks.

3. The OHRP and OCR should work to more clearly distinguish between events that pose physical risks to patients and those that pose informational risks. In doing so, they should clearly delineate which federal regulations apply to each circumstance and ensure that individual research and data collection efforts are not held to multiple conflicting or duplicative regulations.

4. HHS should continue to explore the use of a single, national IRB for multisite studies to ensure more standardized evaluations of projects and more consistent application of regulatory requirements. Reviews are inefficient when conducted by multiple IRBs for multisite studies, adding bureaucratic complexity to the review process and ultimately causing costly delays in the initiation of important projects.

\section{Conclusions}

Federal regulatory agencies need to establish appropriate standards for clinical data collection related to quality improvement that will both adequately protect patients and not unnecessarily inhibit these efforts. The failure of the current regulatory environment to keep up with the science of medicine has resulted in the unnecessary overregulation of research and other data collection efforts that are of minimal risk to the patient. As a consequence, resources are diverted from truly necessary oversight of activities that really do pose credible threats to the welfare of patients.

The urgency of these modifications cannot be overstated. As a result of the Patient Protection and Affordable Care Act of 2010 (Public Law 111-148) and private sector initiatives modeled on federal programs, individual physicians and hospitals are now being held accountable to an unprecedented number of quality reporting mandates (http://sts.org/sites/default/files/documents/ pdf/newsletters/Beltway/HHS-Proposed-RulemakingHuman-Subjects-Research-Protections.pdf). Until more relevant, standardized, and widely disseminated guidance is made available, it is inevitable that inconsistency will persist in local interpretations of the Common Rule and HIPAA Privacy Rule and continue to inhibit our ability to generate quality data on the scale required to drive practice improvements and improve patient care.

\section{Disclosure}

Dr. Asher and Dr. McCormick serve on the board of directors of the NPA. Dr. Glassman is an employee of Norton Healthcare, and has overseen support of non-study related clinical or research effort by Norton Healthcare. Dr. Glassman previously served on the Medtronic corporate advisory board and received consulting fees from Medtronic until July 2011, but is no longer on the board or receives fees. Dr. Glassman has received funds for travel from the Orthopaedic Research and Education Foundation. Dr. Glassman is the president-elect of the Scoliosis Research Society. Rachel
Groman is a paid consultant of Hart Health Strategies and a former employee of the AANS/CNS.

Author contributions to the study and manuscript preparation include the following. Conception and design: Asher, McGirt, Glassman, Resnick, McCormick. Acquisition of data: all authors. Analysis and interpretation of data: Asher, McGirt, Glassman, Resnick, McCormick. Drafting the article: Asher, McGirt, Glassman, Resnick, McCormick. Critically revising the article: Asher, McGirt, Glassman, Resnick, McCormick. Reviewed submitted version of manuscript: Asher, McGirt, Glassman, Groman, Resnick, Mehrlich, Spivey, McCormick. Approved the final version of the manuscript on behalf of all authors: Asher. Administrative/technical/material support: Groman, Mehrlich, Spivey.

\section{References}

1. Ahmed S, Berzon RA, Revicki DA, Lenderking WR, Moinpour CM, Basch E, et al: The use of patient-reported outcomes (PRO) within comparative effectiveness research: implications for clinical practice and health care policy. Med Care [epub ahead of print], 2012

2. Armstrong D, Kline-Rogers E, Jani SM, Goldman EB, Fang J, Mukherjee D, et al: Potential impact of the HIPAA privacy rule on data collection in a registry of patients with acute coronary syndrome. Arch Intern Med 165:1125-1129, 2005

3. Atherton PJ, Sloan JA: Rising importance of patient-reported outcomes. Lancet Oncol 7:883-884, 2006

4. Department of Health and Human Services: Human subjects research protections: enhancing protections for research subjects and reducing burden, delay, and ambiguity for investigators. Fed Regist 76:44512-44531, 2011

5. Deshpande PR, Rajan S, Sudeepthi BL, Abdul Nazir CP: Patient-reported outcomes: a new era in clinical research. Perspect Clin Res 2:137-144, 2011

6. Dokholyan RS, Muhlbaier LH, Falletta JM, Jacobs JP, Shahian D, Haan CK, et al: Regulatory and ethical considerations for linking clinical and administrative databases. Am Heart J 157:971-982, 2009

7. The National Neurosurgery Quality and Outcomes Database ( $\mathrm{N}^{2} \mathrm{QOD}$ ): A Prospective Registry for Quality Reporting. Background, Project Description, Application of Relevant Federal Regulations and Project Implementation. (http://www.neuropoint.org/pdf/N2QOD\%20Project\%20 Description\%20V5\%201-\%20\%20JUL\%202012.pdf) [Accessed November 9, 2012]

8. Patient Protection and Affordable Care Act of 2010, Pub Law 11-148, 124 Stat. 127, Sec. 6301 (March 23, 2010) (http:// www.gpo.gov/fdsys/pkg/PLAW-111publ148/pdf/PLAW111publ148.pdf) [Accessed November 9, 2012]

9. 45 C.F.R. §46. Protection of human subjects. (http://www. access.gpo.gov/nara/cfr/waisidx_06/45cfr46_06.html) [Accessed November 9, 2012]

10. 45 C.F.R. $\$ 160$. General administrative requirements. (http:// www.access.gpo.gov/nara/cfr/waisidx_06/45cfr160_06.html) [Accessed November 9, 2012]

11. 45 C.F.R. \$164. Security and privacy. (http://www.access.gpo. gov/nara/cfr/waisidx_06/45cfr164_06.html) [Accessed November 9,2012]

Manuscript submitted September 14, 2012.

Accepted October 30, 2012.

Portions of this work were presented in abstract form at the AANS meeting in Phoenix, Arizona, in October 2011.

Please include this information when citing this paper: DOI: 10.3171/2012.10.FOCUS12300.

Address correspondence to: Anthony Asher, M.D., Carolina Neurosurgery \& Spine, 225 Baldwin Avenue, Charlotte, North Carolina 28204. email: tony.asher@cnsa.com. 